\title{
From Granular Matter to Generalized Continuum*
}

\author{
J.D. Goddard \\ Department of Mechanical and Aerospace Engineering \\ University of California, San Diego \\ 9500 Gilman Drive \\ La Jolla, CA 92093-0411, USA \\ jgoddard@ucsd.edu
}

Summary. Following a cursory review and synthesis of multipolar continua, the rudiments of graph theory, and granular mechanics, a graph-theoretic, energy-based homogenization is proposed for the systematic derivation of multipolar stress and kinematics in granular media. This provides a weakly non-local hierarchy of multipolar field equations for quasi-static mechanics based on polynomial representations of the kinematics of the type employed in past works. As an improvement on those works, a method is proposed for avoiding "overfitting" of fluctuations based on the so-called "Generalized Additive Method" of statistics. Among other results, it is shown that the standard formula for Cauchy stress in granular media may break down owing to multipolar effects, and that granular rotations in the typical granular medium should not lead to Cosserat effects, as the lowest-order departure from the simple-continuum model.

Key words: granular media, continuum models,homogenization, multipolar, micromorphic, graph theory,polynomial overfitting

\footnotetext{
* In Capriz, G., Giovine, P. and Mariano, P.M., Mathematical Models of Granular Matter, Lecture Notes in Applied Mathematics, Vol. 1937, Chapt. 1, pp. 1-20 , Springer-Verlag, Berlin, 2008.
} 


\section{INTRODUCTION}

Arguments against new ideas generally pass through distinct stages from:

"It's not true" to

"Well it's true but not important" to

"It's true and it's important, but it's not new - we knew it all along"

From The Artful Universe

by John D. Barron

(Chapt. 1 of [16])

This article, an amended and enlarged version of a recent conference paper [21], has its beginnings in a much older work [18] concerned with the largely theoretical question as to the definition of stress in a granular assembly. By no means novel at the time, the question has taken on a more practical importance in the intervening years, in part motivated by "shear bands" associated with the unstable plasticity of granular media. According to simple continuum models, shear bands represent infinitely thin surfaces of discontinuity, in distinct contrast to the zones of finite thickness revealed by numerous experiments and particle-level computer simulations. Moreover, certain of these studies indicate that the grain rotation in shear bands may be quite different from the global rotation (vorticity) outside the band, which is generally interpreted as a manifestation of "Cosserat" effects.

As anticipated in the general field of plasticity and soil mechanics, some type of "enriched" or "structured" continuum model endowed with intrinsic length scale is required to regularize the underlying field equations in the presence of material instability. Furthermore, the additional forces implicated in such models may actually influence the onset and evolution of material instability, as recognized early on by Vardoulakis and coworkers $[36,46]$.

Given the overall progress of granular mechanics in the last two decades, a renewed effort to elucidate the above theoretical questions seems timely and appropriate. With this motivation, the present paper provides a syntheis and critique of various principles and techniques for the homogenization of granular media, with emphasis on the quasi-static mechanical behavior.

A brief review is presented of multipolar continua, regarded as general models for granular media, and a survey is given of the graph-theoretic and energy principles underlying granular micromechanics, based on the interpretation of the associated matrices as differential operators. A novel energybased method is proposed for homogenization, as a modification of the abstract "best fits" proposed elsewhere. This method employs polynomial representations for particle displacements and forces which provide the relevant gradients and moment stresses for micropolar continua. Based on the works of Eringen [16], a general formula is postulated to include contributions from the motion of particle centroids, from particle deformation, and from singular surfaces exhibiting slip or interfacial tension. 
As new results, it is shown that

1. in the absence of intergranular contact moments or external body couples, grain rotations do not contribute directly to the quasi-static stress work, in particular to frictional dissipation, and, therefore,

2. the resultant Cauchy stress derives solely from the motion of particle centroids.

A major goal of the present work is to establish the micropolar continuum as a plausible model for granular and cellular media, by showing in a general and systematic way how micropolar effects emerge from discrete micromechanical models. A second goal is to present a concise formulation of the underlying mathematical techniques, and to connect to basic ideas from topology, graph theory and to other fields of network analysis.

\subsection{Mathematical preliminaries}

The notation is similar to that employed in a previous work [19], where bold symbols are employed for space tensors, lowercase symbols for vectors $\boldsymbol{\varphi}=\varphi^{\alpha} \mathbf{g}_{\alpha}$, uppercase for higher-order tensors $\mathbf{L}=L^{\alpha \beta \cdots} \mathbf{g}_{\alpha} \otimes \mathbf{g}_{\beta} \otimes \ldots$, etc. where $\otimes$ denotes a tensor product, and Greek superscripts and subscripts refer to a basis $\mathbf{g}_{\alpha}, \alpha=1,2,3$, derived from appropriate spatial coordinates. For the present purposes, the latter may be taken as orthogonal cartesian. A colon is employed denote the exhaustive, ordered contractions of tensors of rank two and higher, such that, for $n \geq m,\left(L^{\alpha_{1} \ldots \alpha_{n}}\right):\left(M^{\beta_{1} \ldots \beta_{m}}\right):=$ $L^{\alpha_{1} \ldots \beta_{1}, \ldots \beta_{m}} M_{\beta_{1} \ldots \beta_{m}}$, and we employ superscript $T$ to denote transposition of the right-most tensor component with all the preceding, so that $\left(L^{\alpha_{1} \alpha_{2} \ldots \alpha_{n}} \ldots\right)^{T}:=\left(L^{\alpha_{n} \alpha_{1} \alpha_{2} \ldots} \ldots\right)$. The standard notation $\mathbf{L x}\left(=L_{\beta}^{\alpha} x^{\beta} \mathbf{g}_{\alpha}\right)$ is employed for linear transformations of vectors via second-rank tensors. Roman superscripts are used throughout (in contrast to [21]) to label particles (i.e. grains), branch vectors and the associated graph-theoretical matrices in granular assemblies. Brackets [, ] are employed to denote closed intervals of both reals and integers, and the standard symbol \denotes set exclusion.

With $\mathbf{a}^{n}$ denoting the $\mathrm{n}$-fold symmetric tensor product $\otimes^{n} \mathbf{a}$, the Taylor series expansion for the velocity (or infinitesimal displacement) $\mathbf{v}$,

$$
\mathbf{v}(\mathbf{x})=\mathbf{v}_{o}+\mathbf{L}_{1} \mathbf{r}+\mathbf{L}_{2}: \mathbf{r}^{2}+\ldots,
$$

with

$$
\mathbf{r}=\mathbf{x}-\mathbf{x}_{o}, \mathbf{L}_{n}=\frac{1}{n !}\left(\nabla^{n} \otimes \mathbf{v}\right)_{o}^{T},
$$

provides the well-known expansion for global stress-power density in a simple continuum:

$$
\dot{w}=\frac{1}{V} \int_{V} \mathbf{T}: \mathbf{L} d V=\sum_{n} \dot{w}_{n}
$$

with

$$
\dot{w}_{n}:=\mathbf{T}_{n}: \mathbf{L}_{n}, \quad \mathbf{T}_{n}:=\int_{V} \mathbf{T} \otimes \mathbf{r}^{n} d V,
$$


where $\mathbf{T}$ is Cauchy stress and $\mathbf{L}=(\boldsymbol{\nabla} \otimes \mathbf{v})^{T}$ (first) velocity gradient. With stress moments $\mathbf{T}_{n}$ representing generalized forces conjugate to the kinematical quantities $\mathbf{L}_{n}[19,25]$. Eq. (4), serves to establish an equivalence between a non-homogeneous simple continuum and a homogeneous multipolar continuum i.e. a continuum endowed with intrinsic moment stresses. We distinguish two important special cases:

1. The $\mathbf{L}_{n}$ are identical with higher gradients of the local velocity field, as defined in (2), or

2. they are intrinsic "particulate" fields, say $\mathbf{L}_{n}^{p}(\mathbf{x}, t)$, given by more general constitutive equations.

The first represents a graded (or "Toupin-Mindlin") continuum [23, 25, 35], while the second represents a micromorphic (or "Cosserat-Eringen" ) continuum $[14,24,35,41]^{2}$. Both are endowed with intrinsic length scales, and the graded continuum can be regarded as a manifestation of weak non-locality, a precursor to a fully non-local continuum [17].

By means of the mathematical "fragmentation" of a simple continuum into discontinuous subdomains, Eringen and coworkers have derived micromorphic field theories resembling those obtained by certain statistical mechanical studies of systems of deformable particles [16]. A similar technique has been presented recently for the special case of Cosserat media [13]. The micromorphic continuum is a special case of a multipolar continuum endowed with a polyad of deformable vectors or "directors" attached to each material particle [24], with $\mathbf{L}_{n}^{p}$ representing $3^{n}$ such vectors ${ }^{3}$ The simplest ("grade one") micromorphic continuum is characterized by deformable triad of vectors and, hence, a single second-rank (velocity gradient) tensor $\mathbf{L}^{p}$ attached to each material point that serves to represent an homogeneous microstructural ("particle") deformation and rotation. In the special case of a micropolar (Cosserat) continuum, $\mathbf{L}^{p}=\mathbf{W}^{p}=-\left(\mathbf{W}^{p}\right)^{T}$ and $\boldsymbol{\omega}^{p}=\operatorname{vec}\left(\mathbf{W}^{p}\right)$ represent a (particle) spin generally distinct from the global spin $\mathbf{W}=\left(\mathbf{L}-\mathbf{L}^{T}\right) / 2$. (Recall that [16] distinguishes micropolar as the rigid subclass of microstretch, the isotropic or spherical subclass of micromorphic.)

The various moment stresses may be interpreted, as above, in terms of volumetric working, or alternatively, in terms of their infinitesimal surface actions $\mathbf{T}_{n} \cdot d \mathbf{s}$. Thus, for $n=1$, one has a force ("push" or "pull"), inducing displacement, and for $n=2$, symmetric and skew-symmetric moments (gen-

${ }^{2}$ Both were designated as "micromorphic" in [21], based on the idea that response to higher gradients involves some finite microstructure. Abandoning that unconventional usage, we adopt another, with "graded" designating what is sometime called "gradient" or "higher-gradient" continuum.

3 The basic idea is attributed to Duhem in [44]. Since directors are attached to material points, we exclude from (3) et seq. a term $\dot{w}_{0}=\mathbf{f}_{0} \cdot \mathbf{v}_{0}$ involving a body force $\mathbf{f}_{0}$ and a relative velocity $\mathbf{v}_{0}$, appropriate to two-phase media and anticipated by the theory of mixtures [44]. 
eralized "pinch" $)^{4}$, inducing stretch and rotation, respectively, etc. For later reference, we define a micropolar continuum of grade $m$ as one having $w_{n} \equiv 0$ for $n>m$ for all deformation histories, the simple continuum [43] being grade one.

\subsection{Balances}

According to a microscopic treatment [19], the stress moments $\mathbf{T}_{n}$ in a multipolar continuum should satisfy a hierarchy of balances of the form:

$$
\boldsymbol{\nabla} \cdot \mathbf{T}_{n+1}^{T}+\mathbf{T}_{n}=\mathbf{G}_{n+1}, \text { for } n=0,1, \ldots,
$$

where $\mathbf{T}_{0}:=\mathbf{0}, \mathbf{T}_{1}:=\mathbf{T}$, and the $\mathbf{G}$ 's represent extrinsic body moments plus accumulation of intrinsic multipolar momenta. The latter are not made explicit here, since they are negligible in the quasi-static limit. It is easy to verify that (5) leads to the following integral balance:

$$
\int_{\partial V}\left[\mathbf{T}_{n}+\mathbf{x} \otimes \mathbf{T}_{n-1}+\ldots+\mathbf{x}^{n-1} \otimes \mathbf{T}\right] \cdot d \mathbf{s}=\int_{V} \mathbf{G}_{n} d V, \text { for } n=2,3, \ldots,
$$

in which all stresses up to order $n$ contribute to the $n^{\text {th }}$ surface moment.

The uncertain status of (5) should be acknowledged immediately, since it has not been established by any of the standard methods of continuum mechanics, neither by derivation from the energy balance, by means of invariance principles (objectivity) [15,16,22], nor from variational principles for elastic systems, dating back to the Cosserats $[11,44]$ (and treated in a recent review [30] that includes both micromorphic and graded continua). At any rate, the balance for $n=1$ has the form found elsewhere, up to an arbitrary additive symmetric, second-rank stress [16]). In this author's opinion, the latter might be profitably be regarded as the divergence of a third rank tensor and absorbed into the term $\boldsymbol{\nabla} \cdot \mathbf{T}_{2}^{T}$ in (5).

No attention is paid here to compatibility, discontinuity and boundary conditions for the various kinematic tensors $\mathbf{L}_{n}$, some of which are discussed in [16]. Also, we do not deal with discontinuity and boundary conditions for the associated moment stresses $\mathbf{T}_{n}$, since the main focus of this article is on the issues surrounding the passage from discrete microstructure to continuum model.

\section{MICROMECHANICS}

The kinematics of granular media involves both extrinsic modes or degrees of freedom, associated with motion of particle centroids, and intrinsic or internal modes associated with particle deformation. Although the two are coupled

\footnotetext{
${ }^{4}$ a term suggested by Professor I. Vardoulakis [45].
} 
mechanically through particle contacts, we first focus attention on the extrinsic modes. For the sake of completeness, the following subsection provides some essential background material on granular media $[1,19]$ and elucidates the role of grain rotation.

\subsection{Granular microstructure and rotation}

Fig. 1 illustrates the standard idealization of a granular medium $[19,37]$, with $i, j \in[1, N]$ enumerating particles or grains. With $\mathbf{x}$ denoting the position

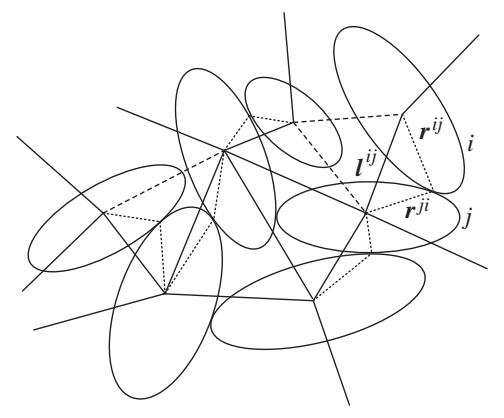

Fig. 1. Idealized granular medium

vector, dotted lines represent moment arms $\mathbf{r}^{i j}=\mathbf{x}^{i}-\mathbf{x}^{i j}$ connecting grain centroid $\mathbf{x}^{i}$ to nominal point of contact $\mathbf{x}^{i j}$, neighbors $j$ being defined by triangulation on centroids (vide infra). Solid and dashed lines then represent branch vectors $\mathbf{l}^{i j}=\mathbf{r}^{i j}-\mathbf{r}^{j i}=-\mathbf{l}^{j i}$, with solid lines indicating active contacts and dashed lines representing virtual contacts (i.e. nearest neighbors without contact).

The interparticle contact force $\mathbf{f}^{i j}$ is the resultant defined by the surface integrals on the left-hand side of (6) with $n=1$, taken over a nominal contact area $i j$. As shown in numerous preceding works, e.g. [1], the assumption of contact forces $\mathbf{f}^{i j}=-\mathbf{f}^{j i}$ localized at points $\mathbf{x}^{i j}$ leads to a particle contribution to the volume-average stress (a "dipole") given by:

$$
\mathbf{T}^{i}=\frac{1}{V^{i}} \sum_{j} \mathbf{f}^{i j} \otimes \mathbf{r}^{i j},
$$

for each particle $i$ in the interior of the granular assembly.

The vector couple about $\mathbf{x}^{i}$ exerted by particle $j$ on $i$ is given

$$
\mathbf{c}^{i j}=\mathbf{f}^{i j} \times \mathbf{r}^{i j}+\mathbf{m}^{i j},
$$

where $\mathbf{m}^{i j}=-\mathbf{m}^{j i}$ is the vector of the skew part of an integral of the type (6), with $n=2$, with $\mathrm{x}$ replaced by $\mathrm{x}-\mathrm{x}^{i}$, and with $\mathrm{x}^{i j}$ representing the 
centroid of the contact stress. Thus, for grains composed of a simple (grade one) material, the contribution from $\mathbf{T}_{2}$ vanishes, so that the contact couple $\mathbf{m}$ arises solely from the moment $\left(\mathbf{x}-\mathbf{x}^{i}\right) \times \mathbf{T} \cdot d \mathbf{s}$ as a kind of rolling resistance.

In the absence of external body couples and contact moments $\mathbf{m}^{i j}$, the quasi-static moment balance requires the symmetry of $\mathbf{T}^{i}$. Furthermore, it is easy to show that

$$
\sum_{i} V^{i} \mathbf{T}^{i}=\sum_{i>j} \mathbf{f}^{i j} \otimes \mathbf{l}^{i j},
$$

mapping particle dipoles into contact dipoles.

On the other hand, the power of internal contact forces is given by the sum over distinct contacts:

$$
\dot{W}=\sum_{i>j} \mathbf{f}^{i j} \cdot \mathbf{v}^{i j}
$$

with

$$
\mathbf{v}^{i j}=\mathbf{u}^{i j}+\boldsymbol{W}^{i} \mathbf{r}^{i j}-\boldsymbol{W}^{j} \mathbf{r}^{j i}, \text { with } \mathbf{u}^{i j}=\dot{\mathbf{x}}^{i}-\dot{\mathbf{x}}^{j},
$$

where $\boldsymbol{W}$ is the skew-symmetric tensor representing particle rotation. Hence, it follows readily that

$$
\dot{W}=\sum_{i>j} \mathbf{f}^{i j} \cdot \mathbf{u}^{i j}+\sum_{i} V^{i} \mathbf{T}^{i}: \boldsymbol{W}^{i}
$$

Since the second term vanishes whenever $\mathbf{T}^{i}$ is symmetric, we have for arbitrary particle shapes the

Theorem In the absence of external body couples and intergranular contact moments $\left(\mathbf{m}^{i j}\right)$ the rotation of internal grains makes no direct contribution to quasi-static stress power.

Here, "internal" refers to those grains in mechanical contact with other grains but not with any boundary from which couples may be transmitted. Of course, the resultant of the latter must be zero.

The preceding theorem has implications for quasi-static Cosserat effects, since most existing micromechanical predictions of such effects, as typified by $[31,33,42]$, depend explicitly on contact moments. However, since the linear dimension of typical (Hertzian) intergranular contact zones, proportional to some $\mathrm{O}(1)$ power of the ratio of confining pressure to elastic modulus, is expected to be small, especially for rigid noncohesive geomaterials such as sand, it follows that the term $\mathbf{f} \times \mathbf{r}$ will dominate the term $\mathbf{m}$ in (8).

The same conclusion results for rigid noncohesive particles with multiple contact zones, since the the contact forces on such zones can be replaced by a finite, statically equivalent set of forces, whose moments are once again captured by the terms of the form $\mathbf{f} \times \mathbf{r}$ in (8). Hence, for nearly rigid grains, any homogenization scheme based on energy principles should yield negligible 
quasi-static Cosserat effects, although particle rotations will generally have other influences on the micromechanics.

It should be noted that certain types of cohesive contacts may give rise to important contact moments, since they allow for a locally large tensile force balanced by a locally large compressive force, producing a large couple $\mathbf{m}$ without a correspondingly large resultant $\mathbf{f}$.

The absence of intergranular contact moments not only justifies various simple-continuum models of granular media but also implies that the breakdown of such models must be due to effects other than those associated with Cosserat rotation. In this connection, we note that the symmetry of (7) and hence of (9), implies that $\mathbf{T}^{T}=\mathbf{T}$ (Cauchy's second law [43]), at least according to the standard formula for $\mathbf{T}$. However, the latter is subject to criticisms presented below.

\subsection{Graph theory for extrinsic modes}

Graph theory provides a particularly appealing tool for the description of various physicochemical networks. In contrast to the mechanical networks associated with structural mechanics [40] or statistical physics [5], the graphs for granular media or other mobile cellular assemblies are often transitory, reflecting abrupt topological rearrangement engendered by finite deformation and requiring a sequence of graphs to describe evolving microstructures. Immediately following is a concise treatment of the graph-theoretical description of granular mechanics, with connections to other fields of application and to the basic mathematical literature.

In a schema dating back to the early works of Satake $[31,37,38]^{5}$, we let particle centroids define the the nodes or vertices $j \in[1, N]$ defined by an appropriate Delaunay triangulation $[1,19,20,37]$. Note that this triangulation should be generally based on (minimal) separation between particle surfaces rather than particle centroids. Whenever unique, this defines an abstract (connected simple) graph $\mathcal{G}$, the granular contact network or Satake graph, with edges or branches $i \in[1, E]$ representing nominal nearest-neighbors and defining contacts or virtual contacts.

In the associated matrix formulation, underlined lowercase quantities denote columns (vertical arrays) associated with edges and nodes, while superscript ${ }^{*}$ denotes transposition (vector-space dual). e.g. $\varphi=\left[\varphi^{i}\right]^{*}=$ $\left[\varphi^{1}, \ldots, \varphi^{N}\right]^{*}$ denotes a $1 \times N$ row of scalars, $\underline{\varphi}=\left[\varphi^{i}\right]^{*}=\left[\varphi^{1}, \ldots, \varphi^{E}\right]^{*}$, a $1 \times E$ row (horizontal array) of space vectors, etc. Then, underlined uppercase denotes the associated linear transformations or matrices, e.g. $\underline{A}=$ $\left[A^{i j}\right], \underline{\mathbf{A}}=\left[\mathbf{A}^{i j}\right]$, etc., with dual or adjoint defined by the standard scalar products $(\underline{u}, \underline{v})=(\underline{v}, \underline{u}):=\underline{u}^{*} \underline{v}$ and $(\underline{\mathbf{u}}, \underline{\mathbf{v}}):=\underline{\mathbf{u}}^{*} \cdot \underline{\mathbf{v}}=\sum_{k} \mathbf{u}^{k} \cdot \mathbf{v}^{k} F$.

\footnotetext{
${ }^{5}$ The present treatment does not rely explicitly on the geometric properties of voids nor on the composite (Schaefer) operators employed in [38] to express micromorphic compatibility, discussed more generally in [16].
} 
Assignment of directions to the edges of the above graph yields a directed graph $[4,6]$, with $E \times N$ incidence matrix $\underline{D}=\left[D^{i j}\right]$ :

$$
D^{i j}=\left\{\begin{array}{c}
+1, \text { if edge } i \text { enters node } j \\
-1, \text { if edge } i \text { leaves node } j \\
0, \text { otherwise }
\end{array}\right.
$$

The matrix $\underline{D}$ (the transpose of the matrix $D$ in $[5,6,38]$ ) and its transpose $\underline{D}^{*}$ represent difference-operators, which we designate, respectively, as the differential and the codifferential (denoted respectively as "coboundary" and "boundary" operators in the standard literature on graph, e.g. p. 5 of [5]). Thus, $\underline{D} \underline{\varphi}$ yields differences along edges of nodal "potentials" represented by $\varphi$, while $\underline{D}^{*} f$ yields nodal accumulations from flows along edges ( $[6]$ and p. 5 of [5]). Since it can be shown that the column rank of $\underline{D}$ is $N-1$, we henceforth delete the final column ${ }^{6}$, corresponding to "ground" node $N$, and denote the resulting $E \times(N-1)$ matrix by the same symbol $\underline{D}$. Accordingly, we dispense with the $N^{\text {th }}$ component of column operands, reducing them to $(N-1) \times 1$ arrays.

Another important operator $\underline{D}_{\times}$is given by

$$
\underline{D}^{*} \underline{D}_{\times}=\underline{0} \text {. and } \underline{D}_{\times}^{*} \underline{D}=\underline{0} \text {, i.e. } \underline{D}_{\times}=\operatorname{ker}\left(\underline{D}^{*}\right),
$$

ker denoting the kernel or null space of a linear transformation. The matrix $\underline{D}_{\times}$can be taken as any $E \times M$ matrix whose $M$ columns form a basis for the null space of $\underline{D}^{*}$, where $M=\operatorname{dim}\left\{\operatorname{ker}\left(\underline{D}^{*}\right)\right\}$, but we shall express it in terms of a normalized cycle basis [4] defined below and denote it as the cross differential of the graph. The operators $\underline{D}, \underline{D}^{*}, \underline{D}_{\times}$then bear an obvious resemblance to grad, div and rot (or curl) of vector calculus, a resemblance made more compelling below.

With power given by $\dot{W}=(f, \underline{u})$, we can formulate a virtual work principle in terms of the above operators as follows (cf. [32]). Designating column $\chi$ as compatible if it can be written as a nodal difference $\chi=\underline{D} \varphi$ and as a conserved flow if it satisfies $\underline{D}^{*} \chi=\underline{0}$, we obtain the associated conservationcompatibility duality $[6,32]$ :

$$
\begin{gathered}
\dot{W}=(\underline{f}, \underline{D} \underline{\varphi})=\left(\underline{D}^{*} \underline{f}, \underline{\varphi}\right)=0 \quad \forall \underline{\varphi}, \text { iff } \underline{D}^{*} \underline{f}=\underline{0} \\
\text { and } \\
\dot{W}=\left(\underline{D}_{\times} \underline{\psi}, \underline{u}\right)=\left(\underline{\psi}, \underline{D}_{\times}^{*} \underline{u}\right)=0 \quad \forall \underline{\psi}, \text { iff } \underline{D}_{\times}^{*} \underline{u}=\underline{0}
\end{gathered}
$$

i.e., compatibility implies conservation and vice versa.

Similar relations apply to arrays of space vector and tensors $\underline{\mathbf{x}}=\left[\mathbf{x}^{i}\right]^{*}$ and $\underline{\mathbf{A}}=\left[\mathbf{A}^{i j}\right]$, with scalar product and adjoint:

$$
(\underline{\mathbf{A}} \underline{\mathbf{y}}, \underline{\mathbf{z}}):=(\underline{\mathbf{A}} \underline{\mathbf{y}})^{*} \cdot \underline{\mathbf{z}}=\left(\underline{\mathbf{y}}, \underline{\mathbf{A}}^{*} \underline{\mathbf{z}}\right) \text {, with } \mathbf{A}^{* i j}=\mathbf{A}^{T j i}
$$

\footnotetext{
${ }^{6}$ not usually done in the prevalent literature and not made explicit in [21].
} 


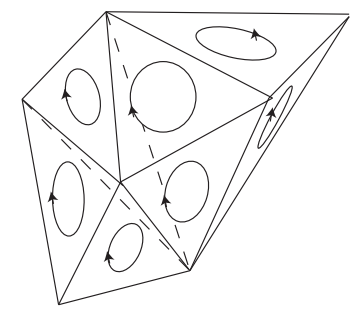

Fig. 2. Meshes for a a $3 \mathrm{~d}$ simplicial complex

Thus, for the Satake graph $\mathcal{G}$, the substitutions $f \rightarrow \underline{\mathbf{f}}$, and $\underline{u} \rightarrow \underline{\mathbf{u}}$ in (15-16) yields the quasi-static equilibrium of forces and the compatibility of relative velocities (or displacements) [31,38]:

$$
\underline{D}^{*} \underline{\mathbf{f}}=\underline{\mathbf{0}}, \text { and } \underline{D}_{\times}^{*} \underline{\mathbf{u}}=\underline{\mathbf{0}}
$$

The second relation of course is satisfied identically by the substitution $\underline{\varphi} \rightarrow \underline{\mathbf{v}}$ in (15-16), where $\underline{\mathbf{v}}=\underline{\dot{x}}$ denotes (nodal) velocities (or infinitesimal displacements) of grain centroids $\underline{\mathbf{x}}=\left[\mathbf{x}^{i}\right]^{*}$, connected by branch or edge vectors $\underline{\mathbf{l}}=\left[\mathbf{l}^{i}\right]^{*}=\underline{D \mathbf{x}}$. The first is satisfied by the substitution in (15-16) of a vector array for a scalar array $\underline{\psi} \rightarrow \underline{\boldsymbol{\psi}}$, providing a discrete analog of the Helmholtz representation of solenoidal vector fields.

The rank of $\underline{D}^{*}$ equals $N-1$ the number of independent scalar balances in the last member of (15) or vector balances in the first member of (18), and the rank of $\underline{D}_{\times}^{*}$ equals $M=E-N+1$, which follows from the celebrated Descartes-Euler polyhedral formula and also from the later analysis of Kirchhoff $[7,29]$ for electrical networks. The latter contains the notion of a cycle basis for conserved flows, which provides a particularly attractive null-space basis for $\underline{D}^{*}$, namely the normalized irreducible cycle basis or mesh for the associated graph $\mathcal{G}$. This consists of the maximal linearly independent set of cyclic currents $f=\left[f_{1}, \ldots, f_{M}\right]^{*}$ having as their only non-zero components $f_{k}$, such that $\left|f_{k}\right|=1$ on branches $k$ forming irreducible (or "elementary" [4]) cycles or "meshes", i.e. cycles that contain no other cycles, on $\mathcal{G}$, as illustrated in Fig. 2 for the polyhedral graph defined by a 3d simplicial complex (i.e. face-connected cluster).

We then take $\underline{D}_{\times}$to be the $E \times M$ matrix (transpose of that denoted by $L$ in [38]):

$$
D_{\times}^{i j}=\left\{\begin{array}{l}
+1, \text { if edge } i \text { is coincident and confluent with cycle } j, \\
-1, \text { if edge } i \text { is coincident and not confluent with cycle } j, \\
0, \text { otherwise }
\end{array}\right.
$$

This imparts the status of difference operator and allows for a symmetric duality in the case of planar graphs, as discussed next. 


\section{A note on duality}

The notion of duality is ubiquitous and varied in the literature on graphs and geometry $[4,12]$, and the following paragraphs represent an attempt to distinguish some special cases particularly relevant to the subject at hand.

In the case of the planar graph $\mathcal{G}$, e.g. associated with planar electrical networks or idealized 2d granular media [31], one can identify a dual graph $\mathcal{G}^{\prime}$ with node-mesh (vertex-face) duality defined by;

$$
M^{\prime}=N-1, N^{\prime}=M+1, E^{\prime}=E
$$

and illustrated by enumerated edges $(e)$, nodes $(n)$ and meshes $(m)$ for the portion of a graph shown in Fig. 3. (The graph on the right is obtained from that on the left by letting the nodes $n$ expand, the meshes $m$ shrink, and the edges $e$ rotate, and vice versa.)
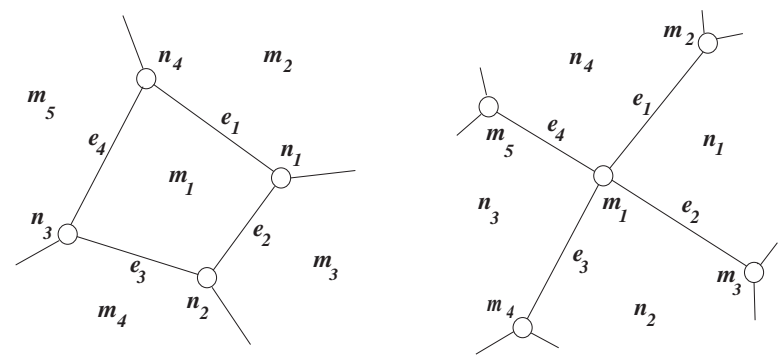

Fig. 3. Node-mesh duality for a planar graph

Fig. 4 illustrates the further grad-rot, force-flow and potential-stream function duality for compatible forces $\underline{u}$ and conserved flows $\underline{f}$ :

$$
\underline{D}^{\prime}=\underline{D}_{\times}, \underline{D}_{\times}^{\prime}=\underline{D}, \underline{u}^{\prime}=\underline{f}, \underline{f}^{\prime}=\underline{u}, \underline{\varphi}^{\prime}=\underline{\psi}, \underline{\psi}^{\prime}=\underline{\varphi},
$$

with

$$
\underline{u}=\underline{D} \underline{\varphi}, \text { and } \underline{f}=\underline{D} \times \underline{\psi}
$$

This highly symmetric duality does not carry over to non-planar graphs such as those associated with $3 \mathrm{~d}$ polyhedra and simplicial complexes, because edges generally are contiguous with more than two faces (or cycles). A duality that preserves edges corresponds to a hypergraph structure [4], with "hyper-edges" connecting more than two nodes. However, another form of duality is possible.

According to a (Schäfli-Poincaré) formula for d-dimensional polytopes (generalized polyhedra) $[12,48]$, we have

$$
\sum_{m=0}^{d-1}(-1)^{m} N_{m}=I_{d}
$$


where $N_{k}$ denotes the number of constituent elements of dimension $k(k=0$ for vertices or nodes, $k=1$ for "edges" connecting vertices, $k \in[2, d]$ for hyperfaces), and $I_{d}$ is a topological invariant depending on the connectivity of the underlying manifold ${ }^{7}$. For simply connected manifolds $I_{d}=1-(-1)^{d}$, giving

$$
N_{0}-N_{1}+N_{2}=2, \quad N_{0}-N_{1}+N_{2}-N_{3}=0,
$$

for $d=3,4$, respectively. The first relation in (24) is the Descartes-Euler polyhedral formula (with $N_{0}=N, N_{1}=E, N_{2}=M$ ), the planar graph representing a $3 \mathrm{~d}$ polyhedral surface, with dual given by (20). With our $3 \mathrm{~d}$ simplicial complex and its graph being regarded as the an appropriate projection of the $4 \mathrm{~d}$ polytope, the second relation in (24) yields a simplex-vertex/edge-face duality:

$$
N_{0}^{\prime}=N_{3}, N_{1}^{\prime}=N_{2}, N_{2}^{\prime}=N_{1}, N_{3}^{\prime}=N_{0}
$$

In the case of granular media composed of convex grains, this is tantamount to the oft-invoked duality [1] between the Voronoi polyhedron centered on a grain and the polyhedral complex of Delaunay simplices having the same center as common vertex. The Voronoi construct can be employed [1] to assign the vectorial area a discussed below to each branch $\mathbf{l}$, which corresponds to the subtended area in [2].

\subsection{Extrinsic power}

As shown above, grain rotation makes no direct contribution to quasi-static work in the absence of body couples and intergranular contact couples. Under these circumstances, the quasi-static power (or incremental work) of contact forces for a granular assembly is given by:
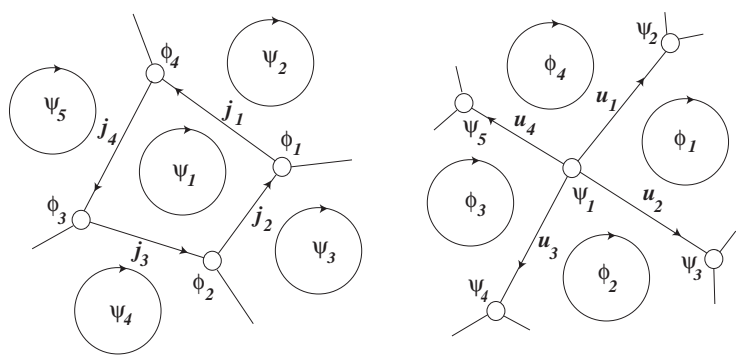

Fig. 4. Potential-function duality

\footnotetext{
7 The actual value of $I_{d}$ is less important for the present purposes than the fact that $\Delta I_{d}=0$ for any addition or deletion of nodes, edges, etc., that preserves connectivity in an existing simplicial complex.
} 


$$
\dot{W}=(\underline{\mathbf{f}}, \underline{\mathbf{u}}):=\underline{\mathbf{f}}^{*} \cdot \underline{\mathbf{u}}=\sum_{k=1}^{E} \mathbf{f}^{k} \cdot \mathbf{u}^{k}
$$

The condition $\dot{W}=0$, analogous to Tellegen's theorem [40] for electrical circuits, requires external forcing of some subset of particles ${ }^{8}$. Otherwise, it represents the virtual-work principle of (15-16).

To pass from contact network (weighted graph) to continuous manifold, we provisionally associate branches $\underline{\mathbf{l}}=\underline{D} \underline{\mathrm{x}}$ with the tangent space, and vectors $\underline{\mathbf{d}}=\left[\mathbf{d}_{k}\right]^{*}, \mathbf{d}^{k}=\mathbf{a}^{k} / V^{k}$, with the cotangent space. As explained below in the Appendix, $\mathbf{a}^{k}$ denotes a vectorial area and $V^{k}=\mathbf{l}^{k} \cdot \mathbf{a}^{k}$ a volume associated with simplicial edge complex (cluster of contiguous Delaunay simplices with common edge $\mathbf{1}^{k}$ ). Thus,

$$
\underline{\mathbf{x}} \Rightarrow \mathbf{x}, \underline{\mathbf{l}}=\underline{D} \underline{\mathbf{x}} \Rightarrow d \mathbf{x}
$$

hence

$$
\underline{D} \varphi=(\underline{D} \underline{\mathbf{x}}, \underline{\mathbf{D}} \varphi) \Rightarrow d \varphi(\mathbf{x})=d \mathbf{x} \cdot \nabla \varphi,
$$

where

$$
\underline{\mathbf{D}}:=\left[\mathbf{d}^{i} D^{i j}\right] \Rightarrow \nabla
$$

The latter is a special case of a higher-order gradient

$$
\underline{\mathbf{D}}^{(n)}:=\left[\mathbf{d}^{i n} D^{i j}\right] \Rightarrow \nabla^{n}, \text { with } \mathbf{d}^{i n}:=\left(\mathbf{d}^{i}\right)^{n}, n=1,2, \cdots,
$$

subject to improvement through the replacement of $D^{i j}$ by a more general (finite-difference) approximation $D^{(n) i j}$, say, based on a connected set of branches. As it stands, (30) is adequate for a continuum interpretation of the results to follow.

\section{ENERGY-BASED HOMOGENIZATION}

In the following, we let $\langle\chi\rangle_{c},\langle\chi\rangle_{\phi}$ denote, respectively, the number averages of the components $\chi^{k}$ of array $\chi=\left[\chi^{k}\right]^{*}$ over branches or edges $k \in[1, E]$ and volume averages over the associated edge complexes, such that

$$
\langle\chi\rangle_{c}:=\frac{1}{E} \sum_{k} \chi^{k},\langle\chi\rangle_{\phi}:=\frac{1}{V} \sum_{k} V^{k} \chi^{k} \text {, and }\langle\chi\rangle_{\phi}=n_{c}\langle V \chi\rangle_{c}
$$

where $n_{c}=E / V$ denotes branch (or total contact) density and $V \chi=\left[V^{k} \chi^{k}\right]^{*}$.

8 The substitution $\dot{W}, \mathbf{v}, \mathbf{u}, \mathbf{f} \rightarrow V, \mathbf{x}, \mathbf{l}, \mathbf{a}$ in (26), a denoting a vectorial area discussed below, yields a geometric formula relevant to granular compaction and dilatancy. By choosing $\mathbf{l}$, a as primary variables in the maximum-entropy estimates discussed in [20], one obtains formulae similar to those proposed in $[8,28]$. 
Then, $\dot{W}$ in (26) may be assumed to arise from boundary forces that provide the stress power:

$$
\dot{w}=\frac{\dot{W}}{V}=\langle\mathbf{T}: \mathbf{L}\rangle_{\phi}=\frac{1}{V} \sum_{k=1}^{E} V^{k} \mathbf{T}^{k}: \mathbf{L}^{k},
$$

the analog of $(3$, where the tensor products $[1,20]$

$$
\mathbf{T}^{k}=\frac{1}{V^{k}} \mathbf{f}^{k} \otimes \mathbf{l}^{k}, \mathbf{L}^{k}=\frac{1}{V^{k}} \mathbf{u}^{k} \otimes \mathbf{a}^{k}, \text { with } V^{k}=\mathbf{l}^{k} \cdot \mathbf{a}^{k},
$$

represent contributions of branches to the global averages $\left\langle\mathbf{T}_{c}\right\rangle,\langle\mathbf{L}\rangle_{c}$ and, hence, to $\left\langle\mathbf{T}_{\phi}\right\rangle,\langle\mathbf{L}\rangle_{\phi}$ As is the case with other heterogeneous media. $\dot{w}$ in (32) generally is not given by $\langle\mathbf{T}\rangle_{\phi}:\langle\mathbf{L}\rangle_{\phi}$, owing to macroscopic gradients and random microscopic fluctuations.

In keeping with the continuum form (1), and following previous works, we assume that $\mathbf{f}^{k}, \mathbf{u}^{k}$ are known, e.g. given by a micromechanical theory or a numerical simulation, and we fit the known data for $\mathbf{u}_{k}$ with

$$
\mathbf{u}^{k}=\tilde{\mathbf{u}}^{k}+\mathbf{u}^{k \prime}, \text { with } \tilde{\mathbf{u}}^{k}=\tilde{\mathbf{L}} \mathbf{l}^{k}+\tilde{\mathbf{L}}_{2}: \mathbf{l}^{k 2}+\ldots+\tilde{\mathbf{L}}_{m}: \mathbf{l}^{k m},
$$

where $\mathbf{l}^{k j}=\left(\mathbf{l}^{k}\right)^{j}$. The polynomial in $\mathbf{l}^{k}$ is attributed to macroscopic gradients and $\mathbf{u}^{k \prime}$ to random fluctuations.

To specify the parameters $\tilde{\mathbf{L}}_{n}$ in (34) for some subset of $m$ branch vectors $\mathbf{l}^{k}$, several authors advocate strict polynomial fits, with a maximal value of $m$, or else some other "best" fit of (34) to continuum kinematics $[27,33,42]$. Given the well-known pathology ("overfitting") of polynomial fits of random data, and in view of the paramount importance of energy, we take the position that a "best" fit should rather be based on minimization of an appropriate norm of stress-power fluctuations plus some norm of the variation implied by (34), in the spirit of the so-called "generalized additive models" (GAM) [49].

As a prototypical linear method, consider

$$
\sigma^{2}=\left(\underline{\mathbf{u}}^{\prime}, \underline{\mathbf{G u}}^{\prime}\right)+Q, \quad \partial \sigma^{2} / \partial \tilde{\mathbf{L}}_{n}=0, n=1,2, \ldots, m
$$

where the $Q$ denotes a quadratic form in the $\tilde{\mathbf{L}}_{n}$. This leads to a set of linear equations for $\tilde{\mathbf{L}}_{n}$, with corresponding estimate for stress power:

$$
\tilde{\dot{w}}=\tilde{\mathbf{T}}: \tilde{\mathbf{L}}+\tilde{\mathbf{T}}_{2}: \tilde{\mathbf{L}}_{2}+\ldots+\tilde{\mathbf{T}}_{m}: \tilde{\mathbf{L}}_{m}
$$

where, as the analog of (4) and in a form proposed elsewhere [19], the moment stresses are given by the average moments (multipoles):

$$
\tilde{\mathbf{T}}_{n}=n_{c}\left\langle\mathbf{f} \otimes \mathbf{l}^{n}\right\rangle_{c}=\frac{1}{V} \sum_{k=1}^{m} \mathbf{f}^{k} \otimes \mathbf{l}^{k n}, n \in[1, m],
$$

irrespective of the resulting solution for $\tilde{\mathbf{L}}_{n}$. 
Although not explored in detail here, one plausible form for $\underline{\mathbf{G}}$ in (35) is

$$
\underline{\mathbf{G}}=\operatorname{diag}\left[\mathbf{1}-\hat{\mathbf{f}}^{k} \otimes \hat{\mathbf{f}}^{k}\right], \text { where } \hat{\mathbf{f}}=\mathbf{f} /|\mathbf{f}|
$$

This penalizes fluctuations $\underline{\mathbf{u}}^{\prime}$ that do no work, representing a loose analogy to thermal fluctuations in molecular systems. One obtains a dual for (34)-(37) by taking $Q$ to be a quadratic form in $\tilde{\mathbf{T}}_{n}$, followed by the interchanges:

$$
\mathbf{u}^{k} \leftrightarrow \mathbf{f}^{k} / V^{k}, n ! \tilde{\mathbf{L}}_{n} \leftrightarrow \tilde{\mathbf{T}}_{n}, \mathbf{l}^{k} \leftrightarrow \mathbf{d}^{k}:=\mathbf{a}^{k} / V^{k}
$$

where $\mathbf{d}^{k n}: \mathbf{l}^{k n}=1$, so that

$$
\tilde{\mathbf{f}}^{k}=V^{k}\left(\tilde{\mathbf{T}} \mathbf{d}^{k}+\tilde{\mathbf{T}}_{2}: \mathbf{d}^{k 2}+\ldots+\tilde{\mathbf{T}}_{m}: \mathbf{d}^{k m}\right)
$$

which corresponds to the so-called "static hypothesis" for contact forces of [10]. The resulting (dual) estimate for $\tilde{\mathbf{L}}_{n}$ is:

$$
\tilde{\mathbf{L}}_{n}=\left\langle\mathbf{L}_{n}\right\rangle=\frac{1}{n !} \sum_{k=1}^{m} \mathbf{u}^{k} \otimes \mathbf{d}^{k n}, n \in[1, m]
$$

with

$$
\underline{\mathbf{L}}_{n}=\frac{1}{n !}\left[\mathbf{u}^{k} \otimes \mathbf{d}^{k n}\right]=\frac{1}{n !} \underline{\mathbf{D}}^{(n)} \otimes \underline{\mathbf{v}},
$$

where $\underline{\mathbf{D}}^{(n)}$ is the matrix defined in (30). The first term in (41) is equivalent to the volume averages couched elsewhere $[1,2]$ in terms of infinitesimal displacement gradients.

Note that, depending on the form of $Q$, the estimates obtained for the moment stresses $\tilde{\mathbf{T}}_{n}$ in (40) would not necessarily agree with those of (37). Similarly, the velocity gradients in (35) are not necessarily the same as those given in (41).

More generally, on replacing $Q$ in (35) by by a quadratic form in both $\tilde{\mathbf{L}}^{k}, \tilde{\mathbf{T}}^{k}$, and employing similar polynomial representations of $\tilde{\mathbf{u}}^{k}, \tilde{\mathbf{f}}^{k}$ in terms of $\mathbf{l}^{k}, \mathbf{d}^{k}$, respectively, we obtain a more general, simultaneous (GAM) estimate of stresses and gradients. Bilinearity of $Q$ in $\tilde{\mathbf{L}}^{k}, \tilde{\mathbf{T}}^{k}$ might allow for an interpretation in terms of energy ${ }^{9}$.

Again, the results for $\tilde{\mathbf{T}}_{n}, \tilde{\mathbf{L}}_{n}$ would not necessarily agree with those obtained above, and it should be amply clear that the definitions of moment stresses and kinematic gradients depend both on the nature of the objective function $Q$ and the inhomogeneity in contact forces and branch vectors.

\subsection{Intrinsic moments and continuum fields}

The preceding discussion deals with extrinsic quantities defined by the motion of grain centroids under the action of intergranular contact forces. The treatment of localized contact mechanics (as in the Hertzian elastic contact), as

\footnotetext{
${ }^{9}$ The preceding paragraph corrects several errors in the corresponding paragraph of $[21]$.
} 
well as the treatment of intrinsic quantities such as global particle deformation would require a consideration of the internal mechanics of individual grains, which one usually assumes to consist of a simple continuum endowed with appropriate constitutive equations, elastic, viscoelastic, elastoplastic, etc.

The detailed treatment of micromechanics is beyond the scope of the present article, which is rather concerned with general aspects and consequences. We merely note that the effective particle stress $\mathbf{T}^{p}$ for a particle $p$ is given by (7), Higher moment stresses are given by a reinterpretation of (37) in which branch vectors $\mathbf{l}$ are replaced by moment arms $\mathbf{r}$. In a similar way, (35) gives a similar but less exact estimate of velocity gradients, by interpreting $\mathbf{u}$ as relative velocity between contact point $k$ and particle centroid, and by basing $\mathbf{d}^{k}$ on an effective contact area. The latter description of particle kinematics represents a type of finite-element approximation, whereas an exact treatment of the micromechanics would generally involve solving field equations for the particle interior, subject to localized tractions on the particle surface, followed by appropriate averaging of solutions over particle volume.

At any rate, it is clear that the localized surface stresses provide a coupling of the intrinsic modes to the extrinsic modes represented by motion of particle centroids. This paramount aspect of granular mechanics may be obscured by the usual micromechanical analysis, where particle rotation, a property of finite grains, is placed $a b$ initio on the same footing as the motion of particle centroids.

With an appropriate replacement of (34) and (35)-(41), one obtains higherorder micromorphic effects, represented by $\mathbf{T}_{n}^{p}, \mathbf{L}_{n}^{p}, n>1$. The ever-increasing dependence on particle length scales is thereby manifest. In a similar vein, we expect that higher-order contact moments will exhibit a similar dependence on the dimension of contact zones.

Given the above estimates of continuum-level moments, the following formula is suggested by, but not rigorously derived from the above-cited works of Eringen and coworkers:

$$
\mathbf{X}=\nu^{c} \mathbf{X}^{c}+\nu^{p} \mathbf{X}^{p}+\mathbf{X}^{s},
$$

where $\mathbf{X}=\left\langle\mathbf{T}_{n}\right\rangle$ or $\left\langle\mathbf{L}_{n}\right\rangle, n=1,2 \ldots$, represent volume (or surface) averages, with $\left\langle\mathbf{X}_{n}\right\rangle^{i}=O(1)$ for for $\nu^{i} \rightarrow 0$. The superscript $c$ refers to a continuumlevel contribution arising from the relative motion of particle centroids; $p$ to a contribution arising from the internal structure of particles, regarded as pieces of a continuous medium; and $s$ to a contribution arising from singular surfaces. $\nu^{p}$ denotes particle volume fraction and $\nu^{c}$ void fraction, given by $1-\nu^{p}$ in the usual granular medium.

Typical singular surfaces involve interfacial slip, such as cracks, or other kinematic discontinuities, or interfacial tension and other (multipolar) stress jumps [16]. The relation (43) appears to cover various limiting case, e.g. $\nu^{p} \rightarrow 0,1$, and Mindlin's special case $\mathbf{X}^{c} \rightarrow \mathbf{X}^{p}$ [35], often used for multipolar elasticity. 
Although the moment stress power is generally not given by $\left\langle\mathbf{T}_{n}^{i}\right\rangle^{i}:\left\langle\mathbf{L}_{n}\right\rangle^{i}$, one may readily obtain the following generalization of a well-known result for $m=1$ from (5): For a graded material of grade $m$, with $\mathbf{L}_{k}^{i}=\left(\nabla^{k} \mathbf{w}\right)^{T} / k$ ! for $k \in[1, m]$, the stress power $\dot{w}_{m}$ is given by

1. $\left\langle\mathbf{T}_{m}\right\rangle: \overline{\mathbf{L}}_{m}$ for velocity fields $\mathbf{w}$ which have boundary values given by a polynomial of maximal degree $m$, with $\overline{\mathbf{L}}_{m}^{i}=\left(\boldsymbol{\nabla}^{m} \mathbf{w}\right)^{T} / m$ !, a constant over $\partial V$, and by

2. $\overline{\mathbf{T}}_{m}:\left\langle\mathbf{L}_{m}\right\rangle$ for moment-stress fields whose surface (moment) tractions satisfy $\mathbf{T}_{k} \mathbf{n}=\delta_{k m} \overline{\mathbf{T}}_{k} \mathbf{n}$, with $\overline{\mathbf{T}}_{m}$ constant over $\partial V$.

Since this result applies to any finite cluster of particles, subject to inhomogeneous conditions of displacement or effective stress on the periphery, one concludes that volume averages do do not provide a proper definition of continuum fields in highly inhomogeneous assemblies. This casts considerable doubt on the use of (7) and, hence, of (9) to define Cauchy stress, the latter of which goes back, in the field of granular mechanics at least to Weber [3,47] and, in theoretical elasticity, to Cauchy, [9] (cf. Note B in the appendix of [34], discussed in [18] and also in [26].). The breakdown of (7) is suggested by the mean-field theory of Jenkins [26] for elastic-sphere assemblies, the theory of Bardet and Vardoulakis [3] for small granular assemblies, and also by more recent calculations [13]. It is worth noting that the theory of [26] involves gradients in contact force reminiscent of (40) above, whereas the other results $[3,13]$ depend on boundary effects in small samples.

\section{CONCLUSIONS}

A synthesis has been presented of graph-theoretic methods and energy-based homogenization to derive continuum models of discrete granular media. As anticipated by several previous workers, it is concluded that the multipolar continuum, either graded or micromorphic, represents a plausible model for the typical granular medium.

It has been shown that the special case of a graded continuum, including the simple (grade one) continuum, is defined solely by the extrinsic modes associated with the motion of grain centroids, in contrast to the micrormorphic continuum, which arises from intrinsic modes represented by the internal mechanics of grains.

Within the subclass of micromorphic continua, the micropolar (Cosserat) limit is appropriate for nearly rigid grains. However, In the absence of intergranular contact moments, it has been demonstrated above that grain rotation makes no direct contribution to quasi-static contact work, and that the widely accepted formula based on volume averaging yields a symmetric Cauchy stress. One therefore concludes that the emergence of Cosserat effects implies the breakdown of this formula. Otherwise, the existence of moment stress must be attribute to kinematic gradients, suggesting that the graded 
continuum may prove to be more appropriate than the micropolar continuum for the quasi-static mechanics of rigid granular media .

There remain open questions as to the validity of the multipolar balances based on [19], the interpretation of Eringen's micromorphic theory in terms of volume averages, and the extension to granular dynamics. Incidentally, given the previous works [16] on micromorphic continua, the latter appears quite feasible.

As pointed out previously [21], further investigations of shear bands and of short-wavelength shear waves should provide a plausible testing ground for multipolar theories.

\section{Acknowledgement}

I am grateful to several colleagues, notably J-J. Moreau, F. Radjai, and N. Rivier, for various discussions of ideas and for bibliographic references. Gratitude is also due to the organizers of the 2005 workshop on granular materials in the Centre Borel, Institut Henri Poincaré, Paris, for providing a tranquil but stimulating environment for some of the effort reflected in this work.

\section{References}

1. K. Bagi. Stress and strain in granular assemblies. Mech. Mater., 22(3):165-177, 1996.

2. J. P. Bardet and J. Proubet. Application of micromechanics to incrementally nonlinear constitutive equations. In J. Biarez and R. Gourvès, editors, Powders and Grains, pages 265-273. Balkema, Rotterdam, 1989.

3. J. P. Bardet and I. Vardoulakis. The asymmetry of stress in granular media. Int. J. Solids Struct., 38(2):353-67, 2001.

4. C. Berge. Graphs and Hypergraphs. North-Holland Publishing Co., Amsterdam, 1973.

5. N. Biggs. Interaction Models. Cambridge University Press,, Cambridge, U.K., 1977.

6. N. Biggs. Algebraic Graph Theory. Cambridge University Press, Cambridge, U.K., 2nd edition, 1994.

7. N. Biggs, E.K. Lloyd, and R.J. Wilson. Graph Theory 1736-1936. Clarendon Press, Oxford, U.K., 1976.

8. R. Blumenfeld and S. F. Edwards. Granular entropy: Explicit calculations for planar assemblies. Phys. Rev. Lett., 90(11):114303/1-4, 2003.

9. A. L. Cauchy. De la pression ou tension dans un système de points matériels. Exércises de maths., 2:42, 1827.

10. C. S. Chang and J. Gao. Kinematic and static hypotheses for constitutive modelling of granulates considering particle rotation. Acta Mech., 115(1-4):213229, 1996. 
11. E. Cosserat and F. Cosserat. Théorie des Corps Déformables (Theory of Deformable Bodies). A. Hermann et Fils, Paris, 1909. (English translation in NASA TT F-475 11,561, Nat. Aero. Space Adm., Washington D.C., February, 1968).

12. H.S.M. Coxeter. Regular Polytopes. Dover, New York, 3rd edition, 1973.

13. W. Ehlers, E. Ramm, S. Diebels, and G. A. D'Addetta. From particle ensembles to Cosserat continua: homogenization of contact forces towards stresses and couple stresses. Int. J. Solids Struct., 40(24):6681-6702, 2003.

14. A. C. Eringen. Theory of micropolar elasticity. In Fracture, an advanced treatise, volume 2, pages 622-728. Academic Press, New York, 1968.

15. A. C. Eringen. Balance laws of micromorphic continua revisited. Int. J. Eng. Sci., 30(6):805-10, 1992.

16. A. C. Eringen. Microcontinuum Field Theories: Foundations and Solids. Springer-Verlag, New York-Berlin, 1999.

17. A. C. Eringen. Nonlocal Continuum Field Theories. Springer,, New York :, 2002.

18. J. D. Goddard. Microstructural origins of continuum stress fields - a brief history and some unresolved issues. In D. De Kee and P.N. Kaloni, editors, Recent developments in sturctured continua, volume 143 of Pitman Research Notes in Mathematics, pages 179-208. Longman/J. Wiley, New York, 1986.

19. J. D. Goddard. Continuum modeling of granular assemblies. In H. J. Herrmann, et al., editor, NATO ASI, Physics of Dry Granular Media, page 24. Kluwer, Dordrecht, 1998.

20. J. D. Goddard. On entropy estimates of contact forces in static granular assemblies. Int. J. Solids Struct., 41(21):5851-61, 2004.

21. J. D. Goddard. Granular media as generalized micromorphic continua. In R. Garcia-Rojo and et al., editors, Powders and Grains 2005, volume 1, pages 129-134. Taylor \& Francis Group, London, 2005.

22. A. E. Green and P. M. Naghdi. A unified procedure for construction of theories of deformable media. 1.classical continuum physics \& 2.generalized continua. Proc. Roy. Soc. London A, 448(1934):335-356,357-377, 1995.

23. A. E. Green and R. S. Rivlin. Multipolar continuum mechanics. Arch. Rat. Mech. Anal., 17(2):113-47, 1964.

24. A. E. Green and S. Rivlin. Relation between director and multipolar theories in continuum mechanics. ZAMP, 18(2):208-18, 1967.

25. A.E. Green and R.S. Rivlin. Simple force and stress multipoles. Arch. Rat. Mech. Anal., 16:325-53, 1964.

26. J. T. Jenkins. Anisotropic elasticity for random arrays of identical spheres. In J. Wu, T. C. T. Ting, and D. M. Barnett, editors, Modern Theory of Anisotropic Elasticity and Applications, pages 368-377. SIAM, Philadelphia, 1990.

27. J. T. Jenkins and M. A. Koenders. The incremental response of random aggregates of identical round particles. Eur. Phys. J. E, 13(2):113-23, 2004.

28. K-I. Kanatani. An entropy model for shear deformation of granular materials. Lett. Appl. Engng. Sci. (Int. J. Eng. Sci.), 18:989-998, 1980.

29. G. Kirchhoff. Üeber die Auflösung der Gleichungen, auf welche man bei der Untersuchung der linearen Vertheilung galvanischer Ströme gefürt wird. Ann. Phys. Chem., 72(12):497-508, 1847. (English translation in Biggs 1976).

30. N. Kirchner and P. Steinmann. A unifying treatise on variational principles for gradient and micro-morphic continua. Phil. Mag., 85:3875-95, 2005. 
31. N. P. Kruyt. Statics and kinematics of discrete Cosserat-type granular materials. Int. J. Solids Struct., 40(3):511-534, 2003.

32. S. Lefschetz. Applications of Algebraic Topology - Graphs and Networks: The Picard-Lefschetz Theory and Feynman Integrals. In Applied mathematical sciences (Springer-Verlag New York Inc.), volume 16. Springer-Verlag,, New York, 1975.

33. C-L. Liao, T-P. Chang, D-H. Young, and C. S. Chang. Stress-strain relationship for granular materials based on the hypothesis of best fit. Int. J. Solids Struct., 34(31-32):4087-100, 1997.

34. A. E. H. Love. A Treatise on the Mathematical Theory of Elasticity. Dover, New York, 4th edition, 1944. (Bibliographic endnotes).

35. R. D. Mindlin. Micro-structure in linear elasticity. Arch. Rat. Mech. Anal., 16(1):51-78, 1964.

36. H. B. Mühlhaus and I. Vardoulakis. Thickness of shear bands in granular materials. Geotechnique, 37(3):271-283, 1987.

37. M. Oda and K. Iwashita, editors. Mechanics of Granular Materials - An introduction. Balkema, Rotterdam/Brookfield, 1999.

38. M. Satake. New formulation of graph-theoretical approach in the mechanics of granular-materials. Mech. Mater., 16(1-2):65-72, 1993.

39. I. V. Savel'ev. Branched coverings over manifolds. J. Math. Sci., 119:605-57, 2004.

40. O. Shai. Deriving structural theorems and methods using Tellegen's theorem and combinatorial representations. Int. J. Solids Struct., 38(44-45 Oct 12):8037$52,2001$.

41. E.S. Suhubi and A. C. Eringen. Nonlinear theory of micro-elastic solids. II. Int. J. Eng. Sci., 2(4):389-404, 1964.

42. A. S. J. Suiker, R. De Borst, and C. S. Chang. Micro-mechanical modelling of granular material. part 1: Derivation of a second-gradient micro-polar constitutive theory. Acta Mech., 149(1-4):161-180, 2001.

43. C. Truesdell and W. Noll. The non-linear field theories of mechanics. In S. Flügge, editor, Encyclopedia of Physics (Handbuch der Physik), volume III/3. Springer-Verlag, Berlin, New York, 1965.

44. C. Truesdell and R. A. Toupin. Principles of classical mechanics and field theory. In S. Flügge, editor, Encyclopedia of Physics (Handbuch der Physik), volume III/1. Springer, Berlin, 1960.

45. I. Vardoulakis. Private communication, 2005.

46. I. Vardoulakis and E. C. Aifantis. Gradient dependent dilatancy and its implications in shear banding and liquefaction. Arch. Appl. Mech. (Ingenieur Archiv), 59(3):197-208, 1989.

47. J. Weber. Recherches concernant le contraintes intergranulaires dans les milieux pulvérents; application à la rhéologie de ces milieux. Cahiers français rhéol., 2:161-170, 1966.

48. E.W. Weisstein. Polyhedral Formula. In http://mathworld.wolfram.com. Wolfram Research Inc.-CRC Press, 2005.

49. S. N. Wood. Stable and efficient multiple smoothing parameter estimation for generalized additive models. J. Am. Stat. Assoc., 99(467):673-86, 2004. 


\section{Appendix: Simplex and edge-complex gradients}

The following, an elaboration on the method employed in [19], serves to define gradients and various geometrical properties associated with simplicial complexes. In a (Euclidean) space of dimension $d$, we define the simplicial gradient of a function $\underline{\varphi}=\left[\varphi^{k}\right]^{*}$, specified on the $d+1$ vertices $\mathbf{x}^{k}$ of a simplex $s$, by means a linear interpolation $\varphi(\mathbf{x})$ based on barycentric coordinates $\xi^{k}(\mathbf{x})[12,32]$ (affine functions of $\mathbf{x}$ defined below ${ }^{10}$ ), with:

$$
\begin{aligned}
& \varphi(\mathbf{x})=\sum_{k=1}^{d+1} \xi^{k} \varphi^{k}, \sum_{k=1}^{d+1} \xi^{k}=1, \quad \xi^{k} \in[0,1], \quad \xi^{k}\left(\mathbf{x}^{k}\right)=1 \\
& \nabla \varphi=\sum_{k=1}^{d+1} \mathbf{g}^{k} \varphi^{k}, \mathbf{g}^{k}:=\nabla \xi^{k} \text { (const.), } \sum_{k=1}^{d+1} \mathbf{g}^{k}=\mathbf{0}
\end{aligned}
$$

so that

$$
\nabla \varphi=\sum_{k=1}^{d+1} \mathbf{g}^{k}\left(\varphi^{k}-\varphi^{o}\right), \quad o \in[1, d+1]
$$

The last member of (45) is merely Green's theorem for simplex $s$, since

$$
\mathbf{0}=\int_{V^{s}} \boldsymbol{\nabla}(1) d V=\int_{\partial V^{s}}(1) d \mathbf{s} \equiv \sum_{k=1}^{d+1} \mathbf{s}^{k}, \text { with } \mathbf{s}^{k}=2 V^{s} \mathbf{g}^{k}
$$

The vector $\mathbf{s}^{k}$ is normal to facet (i.e. a bounding hyperplane of dimension $d-1$ ) $k$, having magnitude $\left|\mathbf{s}^{k}\right|$ equal to its $(d-1)$-volume, and $V^{s}$ is the $d$-volume of the simplex. (The formulae presented here follow from a consideration of the linear map that carries a standard $d$-simplex, i.e. one-half the unit $d$ hypercube, into an arbitrary $d$-simplex.)

Given a basis composed of $d$ edge vectors $\mathbf{g}_{k}=\mathbf{l}^{k}[19]$, an appropriate set $d$ of the $d+1$ vectors $\mathbf{g}^{k}$ provide a reciprocal basis, with $\mathbf{g}^{i} \cdot \mathbf{g}_{j}=\delta_{j}^{i}$. This is illustrated by the special case $\varphi \equiv \mathbf{x}$ in (44), yielding by (46) a well-known expression for the unit tensor:

$$
\mathbf{1}=\sum_{k=1}^{d+1} \mathbf{g}^{k} \otimes \mathbf{g}_{k}, \text { with } \mathbf{g}_{k}=\mathbf{x}^{k}-\mathbf{x}^{o}, \text { for } o, k \in[1, d+1]
$$

In this representation, $\mathbf{x}^{o}$ serves as origin for $\mathbf{g}_{k}, k \in[1, d+1] \backslash o$, with $\mathbf{g}_{j}, j \neq k$, lying in the facet normal to $\mathbf{g}^{k}$. This is illustrated for $d=3, o=4$ in Fig. 5 .

The further special case $\varphi \equiv \xi^{k}$ in (44) yields an explicit formula for barycentric coordinates:

$$
\xi^{k}(\mathbf{x})=\mathbf{g}^{k} \cdot\left(\mathbf{x}-\mathbf{x}^{k}\right)+1, \text { for } k \in[1, d+1]
$$

$\overline{{ }^{10} \xi^{k}=\cos ^{2}} \theta_{k}, \theta_{k} \in[0, \pi / 2]$, provide part of a branched covering [39] of the $(d+1)$ sphere surface by a $d$-simplex. 


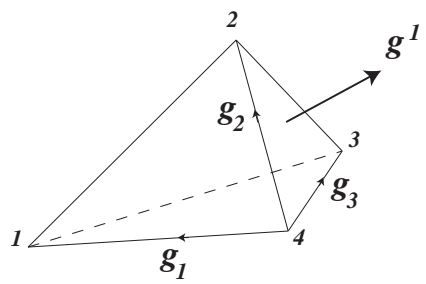

Fig. 5. Elements of an edge basis and its reciprocal facet basis for a $3 \mathrm{~d}$ simplex

with $\xi^{k}\left(\mathbf{x}^{j}\right)=\mathbf{g}^{k} \cdot\left(\mathbf{x}^{j}-\mathbf{x}^{k}\right)+1=\mathbf{g}^{k} \cdot\left(\mathbf{g}_{j}-\mathbf{g}_{k}\right)+1=\delta_{j}^{k}$.

With index $s$ enumerating simplices, (46) becomes

$$
\boldsymbol{\nabla} \varphi^{s}=\sum_{k} \mathbf{g}^{s k}\left(\varphi^{s k}-\varphi^{s o}\right) \equiv \frac{1}{V^{s}} \sum_{k} \mathbf{s}^{s k}\left(\varphi^{s k}-\varphi^{s o}\right)
$$

yielding for the volume-average gradient over an assembly

$$
\langle\boldsymbol{\nabla} \varphi\rangle=\frac{1}{V} \sum_{s} V^{s} \boldsymbol{\nabla} \varphi^{s}=\frac{1}{V} \sum_{s} \sum_{k} \mathbf{s}^{s k}\left(\varphi^{s k}-\varphi^{s o}\right)
$$

where ranges on summations are understood.

For clarity we restrict the discussion to $d \leq 3$ and define a simplicial edge complex (or edge cluster) $\sigma(e)$ to be the set of simplices having common edge $e$, with $e=\{k o\}$ in (50). Then, on rearranging summations and recalling the definition of the matrix differential $\underline{D}=\left[D^{e n}\right]$, we may express (50) as

$$
\langle\nabla \varphi\rangle=\frac{1}{V} \sum_{e=1}^{E} V^{e}\langle\boldsymbol{\nabla} \varphi\rangle^{e}, \text { where }\langle\nabla \varphi\rangle^{e}=\sum_{n=1}^{N} \mathbf{d}^{e} D^{e n} \varphi^{n},
$$

with

$$
\mathbf{d}^{e}=\mathbf{a}^{e} / V^{e}, \mathbf{a}^{e}=\sum_{s \in \sigma(e)} \mathbf{s}^{s} \equiv 2 \sum_{s \in \sigma(e)} V^{s} \mathbf{g}^{e}
$$

The second relation in (51) obviously can be written as $\left[\langle\nabla \varphi\rangle^{e}\right]^{*}=\underline{\mathbf{D}} \underline{\varphi}$, which establishes the relation (29).

The volume $V^{e}$ introduced here is arbitrary and could e.g. be chosen as the sum of simplex volumes $V^{s}, s \in \sigma(e)$. However, for purposes of defining volume averages, it seems more appropriate to employ disjoint volumes, either by reference to particle-based Voronoi cells [1,2] or related particle-free geometric constructs. 